\title{
VISION BASED AUTOMATED ANTHROPOLOGICAL MEASUREMENTS AND ANALYSIS
}

\author{
V. A. Knyaz ${ }^{1,2 *}$, A.A. Maksimov ${ }^{1}$, M.M. Novikov $^{3}$ \\ ${ }^{1}$ State Research Institute of Aviation System (GosNIIAS), 125319 Moscow, Russia - knyaz@gosniias.ru \\ ${ }^{2}$ Moscow Institute of Physics and Technology (MIPT), Russia \\ ${ }^{3}$ Research Center Crystallography and Photonics RAS, Shatura, Russia - novikov@ rambler.ru
}

\section{Commission II, WG II/10}

KEY WORDS: Photogrammetry, 3D reconstruction, photorealistic texturing, accuracy, identification, anthropology

\begin{abstract}
:
Modern techniques of optical 3D measurements such as photogrammetry, computer tomography, laser 3D scanning provide new possibilities for acquiring accurate 2D and 3D data of high resolution, thus creating new conditions for anthropological data analysis. Traditional anthropological manual point measurements can be substituted by analysis of accurate textured 3D models, which allow to retrieve more information about studied object and easily to share data for independent analysis. The paper presents the vision-based techniques for anthropological measurement and investigation needed in various applications, such as morphometric analysis, craniofacial identification, face approximation and other. Photogrammetric methods and their practical implementation in the automatic system for accurate digital 3D reconstruction of anthropological objects are described.
\end{abstract}

\section{INTRODUCTION}

Past decades demonstrated impressive progress and growing availability of new technologies and means for acquiring information of different types about studied object. Such progress provides the condition for putting these new technologies in practice in new research areas where traditional manual tools are mostly utilized. Anthropology and paleoanthropology use visual and geometrical information as an essential data for making decisions on anthropological characteristics of the studied object. And they have long history and rich experience of vision based study of anthropological objects.

Vision based techniques for anthropological study were applied practically at the same time with the photography, when image capturing and recording became available. One of the most famous first vision based anthropological study for person identification was carried out by J. Glaister and J.C. Brash in 1937. They investigated "The Ruxton case" (Glaister and Brash, 1937) by matching the contours of skulls and photographs of two persons and could make a decision about their identity. The technique of superimposition of a person photograph and an image of a skull for making decision about person identification was widely implemented in forensic practice and approved its reliability as forceful anthropological method.

With appearing videocameras, the photo superimposition technique was modified to video superimposition. Now an expert could manually choose the best pose of a skull, looking at live video stream from a camera, on which photograph to be matched is superimposed. This technique has provided an expert some new possibilities for tuning the position and orientation of a skull relative to a given photograph, thus improving the accuracy of the superimposition.

The important part of problems with justification of the superimposition results is related with accurate relative position and

\footnotetext{
${ }^{*}$ Corresponding author
}

orientation of studied photograph and skull, which are essentially the photogrammetric problems. But photogrammetric techniques were introduced in superimposition method not so far ago along with development of digital methods of 3D reconstruction.

Using for analysis an accurate digital 3D model (Weber, 2015) instead a real object has a lot of advantages for researcher such as convenient form of storage, easy reproducing, ability of fast transmitting to desired place of study, possibility of investigation by powerful computer means. Such digital model has to adequately reflect the all essential characteristics of the real object for providing the correctness of the results obtained.

The another approach - facial approximation basing on skull was introduced even before photo superimposition. Notwithstanding that a facial approximation is widely used, the accuracy of face reconstruction is still questionable. So this technique is mostly used as supplementary material in forensic practice.

The paper presents photogrammetric methods and practical system for accurate digital 3D reconstruction of anthropological objects, techniques for accurate photorealistic texturing of the 3D model and algorithms for craniofacial superimposition based on photogrammetric technique.

\section{RELATED WORK}

Person appearance reconstruction basing on skull (or facial approximation) and person identification basing on a skull and a person photograph have a long history of successful development. Facial approximation began with manual plaster reconstruction on real skull (or its plaster copy) (Gerasimov, 1955), (Gerasimov, 1971), (Caldwell, 1986) and now come to intellectual methods of face approximation based on stochastic optimization (Madsen et al., 2018) or based on machine learning on rich demographic information about each subject from large dataset (Booth et al., 2018). Also deep learning models capable of generating object's 
shape 3D reconstruction basing on a single image (Knyaz et al., 2019) appears, which could be applied for facial approximation.

An extensive overview of existing facial approximation (craniofacial reconstruction) techniques considered as a common framework using a general taxonomy is given in (Claes et al., 2010) and (Wilkinson, 2010). The studies show that implementation of the new technologies and the process of permanent evaluating the achieved results and improving the applied techniques provide more and more accurate reconstructions.

Despite the methods of facial approximation became more intellectual and precise, they still could not serve as officially accepted method for obtaining objectively correct human face. Otherwise "craniofacial superimposition has attained a reputation of being a reliable anthropological method, especially for exclusion" (Blau, 2016).

The detailed and comprehensive reviews of historical and methodological development of craniofacial superimposition techniques are given in (Ubelaker, 2015). It reviews the contemporary approaches, including different techniques of digitization (computed tomography, radiography, and ultrasound technology), using of video cameras, warping algorithms, skull positioning devices for reliable superimposition.

The survey (Damas et al., 2011) considers the computer-aided superimposition for forensic identification beginning with determination of the craniofacial superimposition and the description of digital superimposition process and finishing with discussion and existing problems and trends.

\section{CRANIOFACIAL SUPERIMPOSITION}

Craniofacial superimposition (Iscan, 1993) is a forensic process where photographs or video shots of a missing person are compared with the skull that is found. A set of corresponding craniometric and cephalometric landmarks (George, 1993) is typically used for correct superimposition (Table 1 and 2).

\begin{tabular}{|c|c|c|}
\hline \multicolumn{3}{|r|}{ Craniometric Landmarks } \\
\hline Abr & Name & Description \\
\hline $\mathrm{Da}$ & Dacryon & $\begin{array}{l}\text { The point of junction of the frontal, maxillary, and } \\
\text { lacrimal bones on the lateral wall of the orbit. }\end{array}$ \\
\hline Fmt & $\begin{array}{l}\text { Frontomalar } \\
\text { tempora }\end{array}$ & $\begin{array}{l}\text { r The most lateral point of junction of the frontal and } \\
\text { zigomatic bones. }\end{array}$ \\
\hline G & Glabella & $\begin{array}{l}\text { The most prominent point between the supraorbital } \\
\text { ridges in the mid- sagittal plane }\end{array}$ \\
\hline Gn & Gnathion & $\begin{array}{l}\text { A constructed point midway between the most ante- } \\
\text { rior (Pog) and most inferior (Me) points on the chin }\end{array}$ \\
\hline Go & Gonion & $\begin{array}{l}\text { A constructed point, the intersection of the lines tan- } \\
\text { gent to the posterior margin of the ascending ramus } \\
\text { and the mandibular base, or the most lateral point at } \\
\text { the mandibular angle }\end{array}$ \\
\hline $\mathrm{N}$ & Nasion & $\begin{array}{l}\text { The midpoint of the suture between the frontal and } \\
\text { the two nasal bones }\end{array}$ \\
\hline Ns & Nasospinale & $\begin{array}{l}\text { The point where a line drawn between the lower } \\
\text { margins of the right and left nasal apertures is in- } \\
\text { tersected by the midsagittal plane (MSP) }\end{array}$ \\
\hline Pog & Pogonion & $\begin{array}{l}\text { The most anterior point in the midline on the mental } \\
\text { protuberance }\end{array}$ \\
\hline $\operatorname{Pr}$ & Prosthion & $\begin{array}{l}\text { The apex of the alveolus in the midline between the } \\
\text { maxillary central incisor }\end{array}$ \\
\hline Zy & Zygion & The most lateral point on the zygomatic arch \\
\hline
\end{tabular}

Table 1. Craniometric Landmarks

\begin{tabular}{|c|c|c|}
\hline \multicolumn{3}{|r|}{ Cephalometric Landmarks } \\
\hline Abr & Name & Description \\
\hline al & Alare & lateral poi \\
\hline Ec & $\begin{array}{l}\text { Ecto- } \\
\text { canthion }\end{array}$ & $\begin{array}{l}\text { The point at the outer commissure (lateral canthus) } \\
\text { of the palpebral fissure just medial to the malar tu- } \\
\text { bercle (of Whitnall) to which the lateral palpebral } \\
\text { ligaments are attached }\end{array}$ \\
\hline En & $\begin{array}{l}\text { Endo- } \\
\text { canthion }\end{array}$ & $\begin{array}{l}\text { The point at the inner commissure (medial canthus) } \\
\text { of the palpebral fissure }\end{array}$ \\
\hline $\mathrm{g}$ & Glabella & $\begin{array}{l}\text { In the midline, the most prominent point between } \\
\text { the eyebrows }\end{array}$ \\
\hline gn & Gnath & $\begin{array}{l}\text { The point on the soft tissue chin midway between } \\
\text { Pog and Me }\end{array}$ \\
\hline go & Gonion & $\begin{array}{l}\text { The most lateral point of the jawline at the mandibu- } \\
\text { lar angle }\end{array}$ \\
\hline $\mathrm{Me}$ & Ment & est point on the MSP of the soft tissue chin \\
\hline 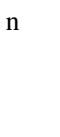 & Nasi & $\begin{array}{l}\text { In the midline, the point of maximum concavity be- } \\
\text { tween the nose and forehead. Frontally, this point } \\
\text { is located at the midpoint of a tangent between the } \\
\text { right and left superior palpebral folds }\end{array}$ \\
\hline pog & Pogo & The most anterior point of the soft tissue chin \\
\hline $\mathrm{Li}$ & $\begin{array}{l}\text { Labiale } \\
\text { inferius }\end{array}$ & The midpoint on the vermilion line of the lower lip \\
\hline Ls & $\begin{array}{l}\text { Labiale } \\
\text { superius }\end{array}$ & The midpoint on the vermilion line of the upper lip \\
\hline sn & Subnasale & $\begin{array}{l}\text { The midpoint of the columella base at the angle } \\
\text { where the lower border of the nasal septum meets } \\
\text { the upper lip }\end{array}$ \\
\hline $\mathrm{t}$ & Tragion & $\begin{array}{l}\text { Point in the notch just above the tragus of the ear; it } \\
\text { lies } 1 \text { to } 2 \mathrm{~mm} \text { below the spine of the helix, which } \\
\text { can be palpated }\end{array}$ \\
\hline $\mathrm{Zy}$ & Zygion & $\begin{array}{l}\text { The most lateral point of the check (zygomaticoma- } \\
\text { lar) region }\end{array}$ \\
\hline
\end{tabular}

Table 2. Cephalometric Landmarks

Usually the landmarks are chosen so that the thickness of the soft tissue is relatively low. The reason for such choice is the necessity make decision in case when there can be changes in age and weight of a person.
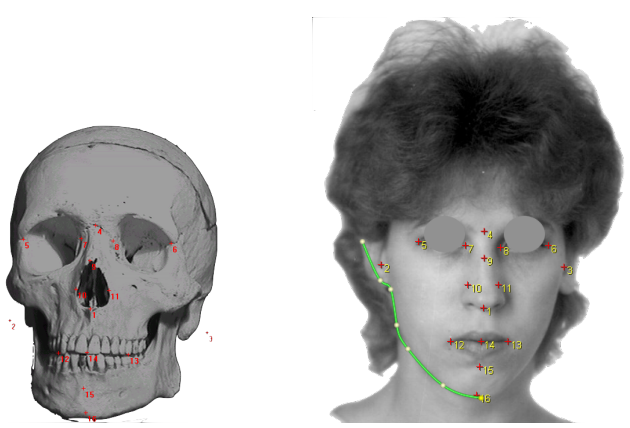

Figure 1. Craniometric and cephalometric landmarks

The analysis performed in (Damas et al., 2011) allowed to indicate as the key problem of reliable craniofacial superimposition the orientation problem: to find "the size and orientation of the skull to correctly match the head in the photo". This problem is essentially photogrammetric one, but the photogrammetric approach did not widely used in a practice as a basis for the problem solution. Most part of techniques use directly acquired a skull photo and then aligning it with a given face photograph using commercial (e.g. Adobe Photoshop) or original software (Scully and Nambiar, 2002), (Ghosh and Sinha, 2005). In fact, it would be most of the times impossible to obtain the technical specifications used to take the antemortem photograph. 


\section{PHOTOGRAMMETRIC SYSTEM}

The task of craniofacial identification is to answer to the question if the given skull is the skull of the person imaged on given photograph, having a real skull and a photograph with (typically) unknown image orientation parameters. In forensic the decision is made basing on quality of a correspondence of a set of craniometric points to a set of cephalometric points of the face imaged at a photograph and a correspondence of a set of contours of the skull and the photograph.

Another (photogrammetric) words the task is the image orientation task having a real object and (probably) its image. The quality of orientation serves as criterium of correspondence.

Current stage of development of photogrammetric means and methods allow to create accurate textured 3D models, which have all geometric and texture characteristics of a real object (Knyaz et al., 2018). So the proposed approach for cranifacial identification based on creating an accurate skull 3D model, mapped with photorealistic texture. Photorealistic texture is generated from a set of skull images with known orientation.

To implement this approach an automated PC-based digital photogrammetric system is designed. It started from automatic photogrammetric system for skull 3D reconstruction (Knyaz et al., 2000). Now it provides all functions needed for cranifacial identification, beginning with system calibration and 3D model generation and completing with forensic report generation.

\subsection{System configuration}

Photogrammetric system is designed as fully automated device for textured skull 3D model generation (Figure 2). It includes:

\section{- 4 high resolution cameras}

- precise PC-controlled rotation stage

- 2 laser line source

- shadow-free light sources for texture generation

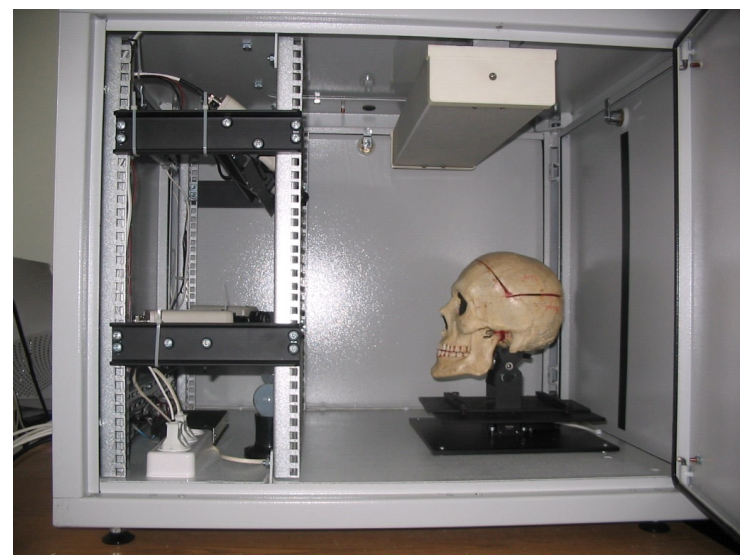

Figure 2. Automated digital photogrammetric system

Such configuration allows to obtain accurate skull 3D model in automatic mode and perform accurate texture mapping.

\subsection{Calibration}

Non-metric cameras equipped with wide angle lens $(6 \mathrm{~mm}$ focal length) are used for image acquisition, thus captured images have a significant distortion. Original automatic calibration procedure estimates image interior orientation parameters and provides high accuracy of 3D reconstruction and texture mapping.

Preliminary calibration includes estimation of cameras interior and exterior orientation and the orientation of rotation stage axis. Calibration process is fully automated due to applying PC-controlled positioning device (rotation stage) and original coded targets for reference points marking (Figure 3).

The test field for system calibration is a precise plane plate with 49 reference points. It determine the system of coordinates in which skull 3D model is generated and in which external orientation of acquired images are determined.

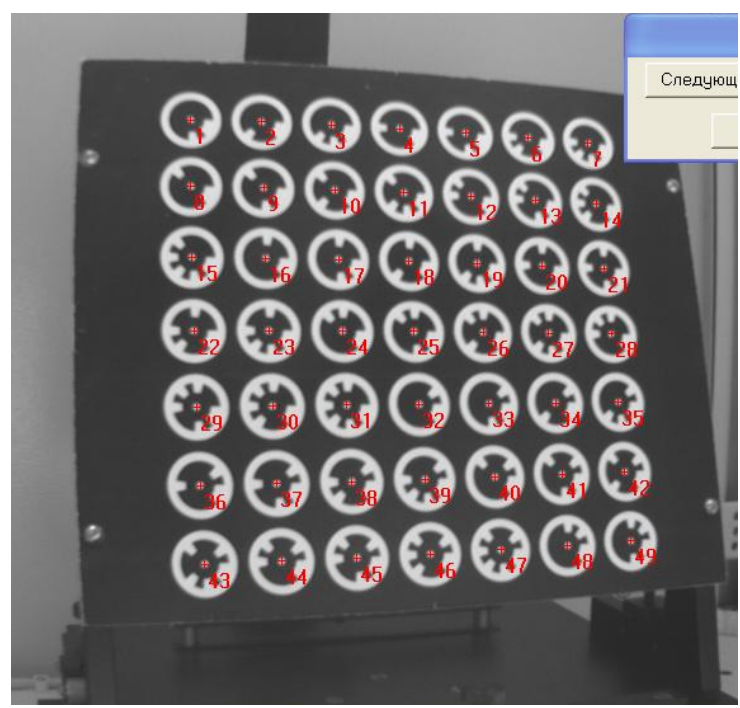

Figure 3. Rotation stage with test field for calibration procedure

The calibration procedure starts from the basic model of image formation - the collinearity equation, expressing the condition that the point of the scene $G$, the center of the projection of $O$ and the image of this point $g$ lie on one straight line:

$$
\mathbf{X}_{G}=\mathbf{X}_{0}-\mu \mathbf{A}^{T} \cdot\left(\mathbf{x}_{g}-\mathbf{x}_{p}\right)
$$

Here

$\mathbf{X}_{0}=\left(X_{0}, Y_{0}, Z_{0}\right)-$ coordinates of the center of the projection, $\mathbf{X}_{G}=(X, Y, Z)$ - scene point coordinates,

$\mathbf{x}_{g}=(x, y,-f)-$ the corresponding coordinates of the scene point in the image,

A - coordinate system transformation matrix,

$\mathbf{x}_{p}$ - coordinates of the main point of the snapshot,

$\mu-$ scale factor.

To account for distortions, additional terms are introduced into the collinearity equations $\Delta x$ and $\Delta y$, describing various distorting factors. Then the real (distorted) $x_{d}, y_{d}$ coordinates of a point in the image are defined as: 
The practice of photogrammetric measurements have proved that the good description for nonlinear distortion is the following model (Beyer, 1992):

$$
\begin{aligned}
\Delta x & =a_{0} \cdot y_{a}+x_{a}\left(a_{1} r^{2}+a_{2} r^{4}+a_{3} r^{6}\right) \\
& +a_{4}\left(r^{2}+2 x_{a}^{2}\right)+2 a_{5} x_{a} y_{a} ; \\
\Delta y & =a_{0} \cdot x_{a}+y_{a}\left(a_{1} r^{2}+a_{2} r^{4}+a_{3} r^{6}\right) \\
& +a_{5}\left(r^{2}+2 y_{a}^{2}\right)+2 a_{4} x_{a} y_{a} ;
\end{aligned}
$$

with $r^{2}=x_{a}^{2}+y_{a}^{2}$

Here

$x_{a}, y_{a}$ - coordinates of a point on the image,

$a_{0}, \ldots, a_{5}$ - camera interior orientation parameters:

$a_{0}$ - coefficient of affine distortion;

$a_{1}, a_{2}, a_{3}$ - coefficients of radial distortion;

$a_{4}, a_{5}$ - coefficients of tangential distortion.

The vector $v_{e}^{l}=\left(x_{p}, y_{p}, m_{x}, m_{y}, a_{0}, \ldots, a_{5}\right)^{T}$ of estimated parameters for test field calibration includes coordinates of principal point, image scales and additional parameters correspondingly, spatial coordinates of reference points being known by independent precise measurements.

The results of calibration procedure are presented in Table 3.

\begin{tabular}{|c|ll|ll|}
\hline \multicolumn{5}{|c|}{ Camera rmse, mm } \\
\hline \hline & \multicolumn{2}{|c|}{ Lower } & \multicolumn{2}{c|}{ Upper } \\
\hline & Left & Right & Left & Right \\
\hline$\sigma_{x}$ & 0.022 & 0.024 & 0.026 & 0.026 \\
$\sigma_{y}$ & 0.023 & 0.021 & 0.023 & 0.025 \\
$\sigma_{z}$ & 0.033 & 0.035 & 0.037 & 0.039 \\
\hline \hline \multicolumn{5}{|c|}{ Max error, mm } \\
\hline$\delta$ & 0.083 & 0.078 & 0.087 & 0.092 \\
\hline
\end{tabular}

Table 3. Results of system calibration

Table 3 ensures the accuracy of the generated 3D models at the level of $0.05 \mathrm{~mm}$. This accuracy is sufficient for the task of anthropological analysis.

The textured digital model provides the expert with more information, as some features can only be found on the color image of the object. Since the photogrammetric system has been calibrated for all three cameras using a single calibration field, this ensures that the texture is accurately superimposed on the geometric coordinates of the digital model.

\subsection{Rotation stage axis determination}

Another suggestion used for 3D reconstruction is the following: contrast line found in the image is the intersection of light plane with object which rotates with given step around given axis. To obtain metric characteristic of reconstructed 3D model the axis of rotation should be known with high precision. The estimation of rotation axis is based on image acquisition of plane test field in $n$ several positions while rotating the turntable.

Then spatial coordinates of reference points are calculating basing on known exterior orientation of cameras for each angle position $\phi_{i}, i=1,, n$ of test field. The vector of unknown parameters for least mean squares estimation includes rotation axis orientation $\left(X_{a}, Y_{a}, Z_{a}, \alpha_{a}, \omega_{a}, \kappa_{a}\right)$ and angles of rotation $\phi_{i}, i=$ $1,, n$.

\begin{tabular}{cccccc}
\hline \hline $\begin{array}{c}X a, \\
m m\end{array}$ & $\begin{array}{c}Y a, \\
m m\end{array}$ & $\begin{array}{c}Z a, \\
m m\end{array}$ & $\begin{array}{c}\alpha_{a}, \\
\text { rad }\end{array}$ & $\begin{array}{c}\omega_{a}, \\
\text { rad }\end{array}$ & $\begin{array}{c}\kappa_{a}, \\
\text { rad }\end{array}$ \\
\hline-0.35 & 0.0 & -2.02 & 0.0 & 0.0059 & -0.0043 \\
\hline \hline
\end{tabular}

Table 4. Results of rotation axis calibration

The results of estimation for rotation axis orientation are given in Table 4. They show that rotation axis is near the $Y$ axis of external coordinate system and has a shift in $X, Z$ direction which has to be accounted for $3 \mathrm{D}$ coordinates calculating.

\section{3D MODEL RECONSTRUCTION AND SUPERIMPOSITION}

Skull 3D model is generated as a set of spatial point arrays obtaining by laser line projection on a skull matching in left and right images at each position of the rotation stage. Then triangulation of $3 \mathrm{D}$ points is performed for complete surface reconstruction.
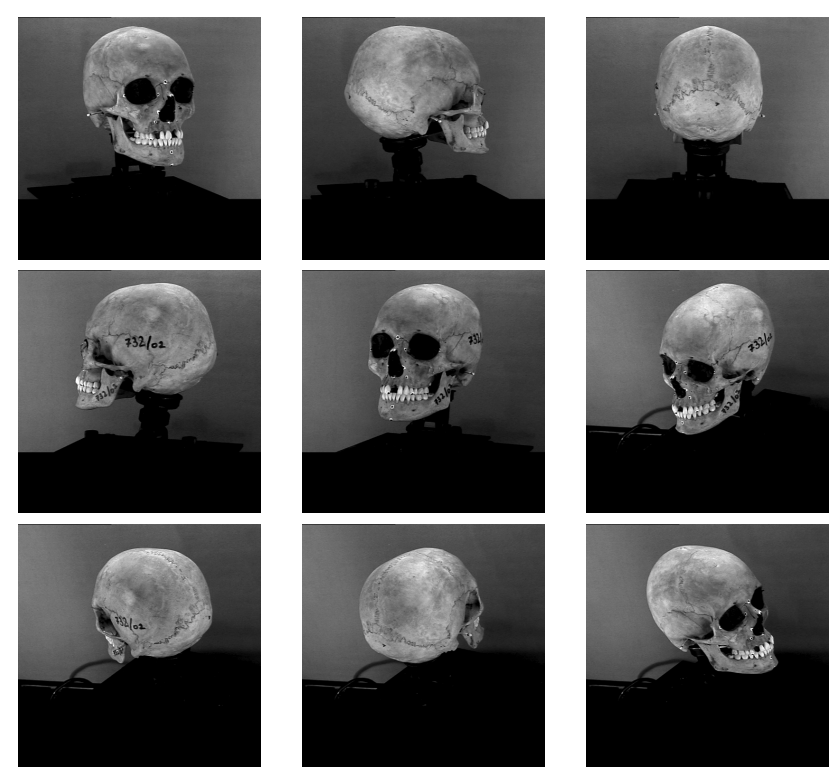

Figure 4. A set of texture images.

Because of complicated topology of a skull a set of images in shadow-free light is needed for accurate texture generation. To provide metric texture mapping for reconstructed 3D model nine images are captured from as lower so upper cameras at given positions of the rotation stage (Figure 4).
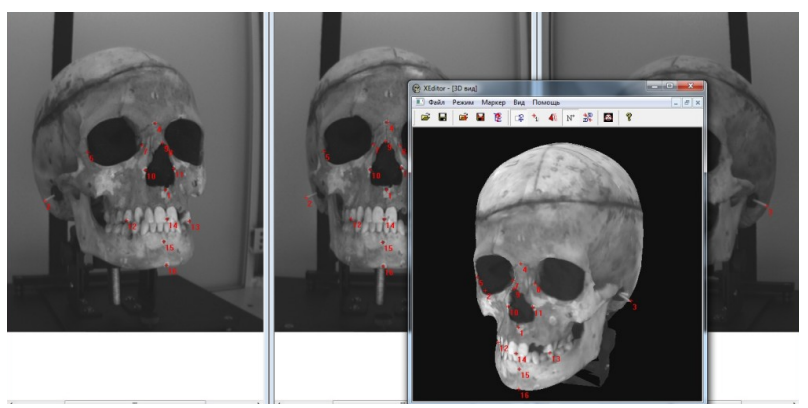

Figure 5. Craniometric landmarks maesurement

Figure 5 shows the resulting textured skull 3D model along with three images acquired for determination of $3 \mathrm{D}$ coordinates of 
craniometric landmarks.

A special procedure is developed for determination of 3D coordinates of craniometric landmarks. An expert indicates needed landmarks in the textured skull 3D model using an original software of the photogrammetric system. Then these $3 \mathrm{D}$ points are projected into three images of a skull - left, frontal and right (Figure 5).

The expert can correct image coordinates of the landmarks using the three images, thus providing the higher accuracy of the landmarks 3D coordinates. The residuals at the landmark points are shown to an expert as an index of 3D coordinates accuracy.

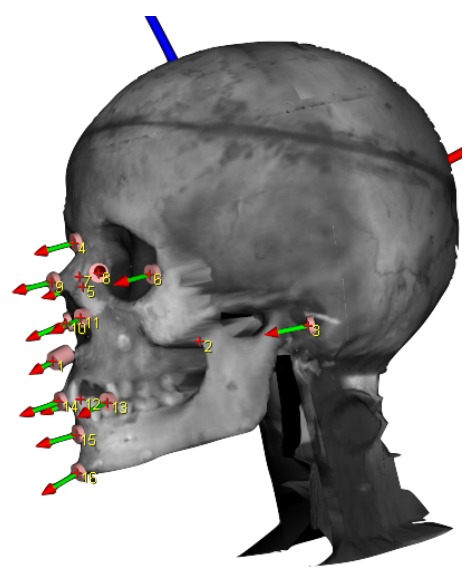

Figure 6. Soft tissue thickness accounting

The process of craniofacial superimposition is considered as the process of photograph external orientation basing on a set of 3D landmark point, obtained at the previous stage. To account for tissue a set of vectors $v_{t}^{i}$ introduced (Figure 6). The value of vector $\left|v_{t}^{i}\right|$ is a value of tissue thickness at the landmark point $l^{i}$, the direction of the vector $v_{t}^{i}$ is the direction from a craniometric landmark to corresponding cephalometric landmark.

Photograph exterior orientation is performed by least mean square estimation using craniometric landmarks (corrected for tissue thickness) as reference points. The residuals at landmark points are treated as a quality of superimposition and a particular index of skull-photograph correspondence.

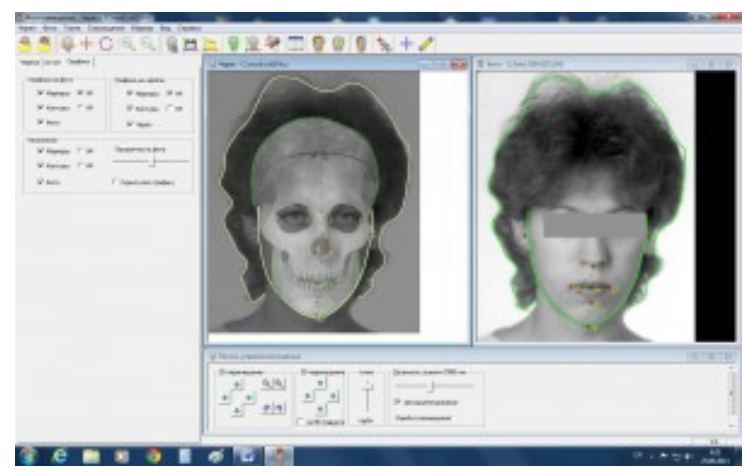

Figure 7. Identification process

Figure 7 presents a sample of superimposition using this technique. After image orientation an expert investigates face contours correspondence.

\section{CONCLUSION}

Photogrammetric system for accurate 3D reconstruction of anthropological objects is developed. It allows to create digital 3D models of high resolution in fully automatic mode. Accurate photorealistic texturing based on precise image orientation and photometric image correction provides an expert with consistent $2 \mathrm{D}$ and 3D data, which is necessary for comprehensive anthropological measurements and analysis.

Original automatic calibration procedure assures the high accuracy of 3D reconstruction and accurate photorealistic texture mapping of the created 3D models. These textured 3D models allow easily to find necessary anthropological landmarks and measure their spatial coordinates.

The developed algorithms solve the problem of craniofacial superimposition as an exterior orientation problem of a person photograph. 3D coordinates of craniometric landmarks, obtained from accurate skull 3D model, serves as a reference points for corresponding cephalometric landmarks measured in the given photograph. Such photogrammetric technique allow to achieve high quality of superimposition needed for reliable craniofacial identification.

\section{ACKNOWLEDGEMENTS}

The reported study was funded by Russian Foundation for Basic Research (RFBR) according to the research project 17-29-04509.

\section{REFERENCES}

Beyer, H., 1992. Advances in characterization and calibration of digital imaging systems. Int. Arch. Photogramm. Remote Sens. Spatial Inf. Sci. XXIX, pp. 545-555.

Blau, J., 2016. Handbook of Forensic Anthropology and Archaeology. WAC Research Handbooks in Archaeology, Taylor $\&$ Francis.

Booth, J., Roussos, A., Ponniah, A., Dunaway, D. and Zafeiriou, S., 2018. Large scale 3d morphable models. International Journal of Computer Vision 126(2), pp. 233-254.

Caldwell, M. C., 1986. New questions (and some answers) on the facial reproduction techniques. Charles C. Thomas, Springfield, IL, pp. 229-255.

Claes, P., Vandermeulen, D., Greef, S. D., Willems, G., Clement, J. G. and Suetens, P., 2010. Computerized craniofacial reconstruction: Conceptual framework and review. Forensic Science International 201(1), pp. 138 - 145. Meeting of the International Organization of Forensic Odonto-Stomatology.

Damas, S., Cordón, O., Ibáñez, O., Santamaría, J., Alemán, I., Botella, M. and Navarro, F., 2011. Forensic identification by computer-aided craniofacial superimposition: A survey. ACM Comput. Surv. 43(4), pp. 27:1-27:27.

George, R., 1993. Anatomical and artistic guidelines for forensic facial reconstruction. Wiley, New York, pp. 215-227.

Gerasimov, M., 1955. Vosstanovlenie lica to cerepu. Moskva: Izdat. Akademii Nauk SSSR. In Russian.

Gerasimov, M., 1971. The face finder. London: Hitchinson \& Co. 
Ghosh, A. and Sinha, P., 2005. An unusual case of cranial image recognition. Forensic Science International 148(2), pp. $93-100$.

Glaister, J. and Brash, J., 1937. THE MEDICO-LEGAL ASPECTS OF THE RUXTON CASE. E. \& S. Livingstone, Edinburgh.

Iscan, M., 1993. Introduction to techniques for photographic comparison. Wiley, New York, pp. 57-90.

Knyaz, V. A., Kniaz, V. V. and Remondino, F., 2019. Imageto-voxel model translation with conditional adversarial networks. In: L. Leal-Taixé and S. Roth (eds), Computer Vision-ECCV 2018 Workshops, Springer International Publishing, Cham, pp. 601-618.

Knyaz, V. A., Leybova, N. A., Galeev, R., Novikov, M. and Gaboutchian, A. V., 2018. Photogrammetric techniques for paleoanthropological objects preserving and studying. ISPRS - International Archives of the Photogrammetry, Remote Sensing and Spatial Information Sciences XLII-2, pp. 525-530.

Knyaz, V., Zheltov, S. and Stepanyants, D., 2000. Automated photogrammetric system for photorealistic skull 3d reconstruction. Proc. SPIE, Videometrics and Optical Methods for 3D Shape Measurement. 4309, pp. 4309-1 - 4309-10.

Madsen, D., Lthi, M., Schneider, A. and Vetter, T., 2018. Probabilistic joint face-skull modelling for facial reconstruction. In: The IEEE Conference on Computer Vision and Pattern Recognition $(C V P R)$.

Scully, B. and Nambiar, P., 2002. Determining the validity of furue's method of craniofacial superimposition for identification. Annals of Dentistry University of Malaya 9(1), pp. 17-22.

Ubelaker, D. H., 2015. Craniofacial superimposition: Historical review and current issues. Journal of Forensic Sciences 60(6), pp. 1412-1419.

Weber, G. W., 2015. Virtual Anthropology and Biomechanics. Springer Berlin Heidelberg, Berlin, Heidelberg, pp. 937-968.

Wilkinson, C., 2010. Facial reconstruction-anatomical art or artistic anatomy? Journal of anatomy 216(2), pp. $235-250$. 